\title{
CyberKnife Stereotactic Radiotherapy in Secondary Vasoproliferative Tumor of the Retina
}

\author{
Şefik Can İpek ${ }^{a} \quad$ Murat Tunç $^{b}$ Kaan Oysulc Aylin Yaman ${ }^{d}$ \\ Ali Osman Saatci ${ }^{\mathrm{T}}$ \\ aDepartment of Ophthalmology, Ağrı Research and Training Hospital, Ağrı, Turkey; \\ ${ }^{b}$ Department of Ophthalmology, Tunç Eye Clinic, Ankara, Turkey; 'Department of Radiation \\ Oncology, Medicana Hospital, Ankara, Turkey; ${ }^{\mathrm{d} D e p a r t m e n t ~ o f ~ O p h t h a l m o l o g y, ~ D o k u z ~ E y l u l ~}$

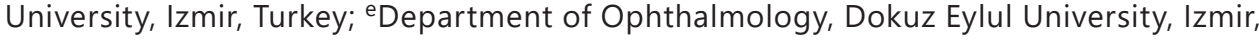 \\ Turkey
}

\section{Keywords}

Stereotactic radiotherapy $\cdot \mathrm{X}$-linked retinoschisis $\cdot$ Vasoproliferative tumor of the retina

\begin{abstract}
A 16-year-old boy with X-linked retinoschisis was examined for the visual decline on his left eye. Upon examination, a vasopermeable elevated mass lesion was determined at the lower temporal retinal periphery associated with surrounding arc-like hard exudates which is consistent with a vasoactive proliferative tumor of the retina. After discussing the therapeutic options with the patient and his parents, 3 successive dexamethasone implants 6 months apart were administered. As no satisfactory regression was noticed, CyberKnife stereotactic radiosurgery (SRS) was performed. Regression of the exudative tumor was obtained in follow-up examinations at 12 and 24 months after the SRS session and the thickness of the lesion was markedly reduced.
\end{abstract}

(C) 2021 The Author(s).

Published by S. Karger AG, Basel

\section{Introduction}

Vasoproliferative tumors of the retina (VPTR) are rare lesions which were initially termed as presumed acquired retinal hemangiomas $[1,2]$. They may be classified as primary (75\% of cases) or secondary due to various ocular conditions (25\%) including X-linked juvenile retinoschisis (XLRS), which is a genetic entity occurring by mutations in the retinoschisin 1 (RS1) gene [2-4]. Currently, there is no approved effective treatment for the management of VPTR $[1,2]$. We hereby present a case with XLRS and unilateral vasopermeable 
lesion, consistent with VPTR which was initially treated by 3 successive intravitreal dexamethasone implants and subsequently with CyberKnife stereotactic radiosurgery (SRS) to control the associated severe retinal exudation progressively impairing the vision.

\section{Case Presentation}

A 16-year-old boy who had a previous history of XLRS was examined for a gradual visual decline on his left eye. He has been on topical brinzolamide 3 times a day for 2 years. On eye examination, his best-corrected visual acuity was 3/10 in OD and 2/10 in OS. The slit lamp examination was unremarkable. Intraocular pressures were $15 \mathrm{~mm} \mathrm{Hg}$, OU. There was a spoke-wheel like appearance at the right posterior pole (Fig. 1a), and the optical coherence tomography exhibited an inner retinal splitting (Fig. 1b). There was an arc-like exudative accumulation surrounding the highly elevated mass at the lower temporal retinal periphery in OS (Fig. 1c). Optical coherence tomography exhibited inner retinal splitting with extensive subretinal fluid temporal to the macula in OS (Fig. 1d).There was no peripheral retinoschisis or any sign of exudation in the right eye. No macular leakage was present in the right eye angiographically as expected. The mass lesion was hypofluorescent throughout the fluorescein angiogram with hyperfluorescent vascular dilatations at the stained lesion border consistent with VPTR in OS (Fig. 1e-g).

The subretinal exudation was detected around the tumor in OS. Therapeutic options were discussed with the parents and 3 intravitreal dexamethasone implants were administered in a time frame of 18 months almost 6 months apart. Although 3 consecutive dexamethasone

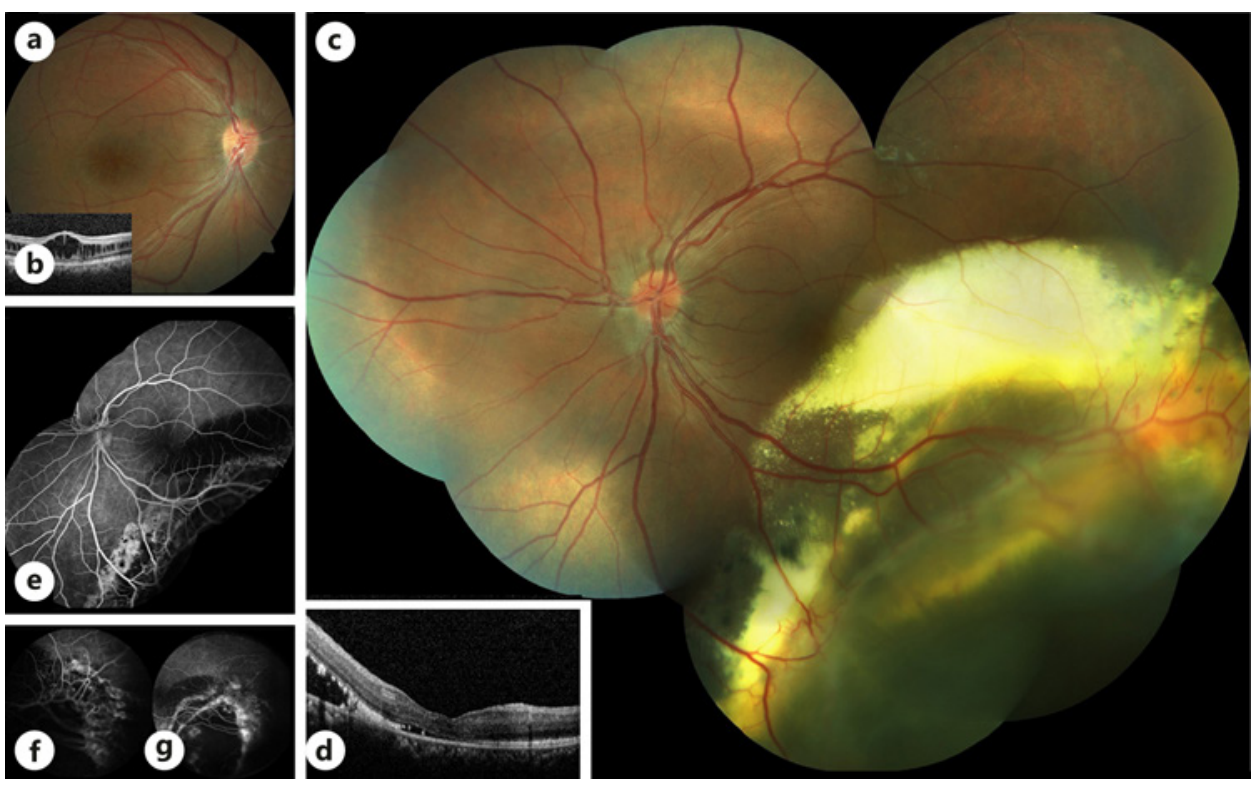

Fig. 1. At the time of initial presentation. a Color fundus picture of the right eye; spoke-wheel like appearance of the fovea. $\mathbf{b}$ Optical coherence tomographic section of the right eye; inner retinal splitting. c Composite color fundus picture of the left eye; temporally located elevated mass-like lesion with the arc-like hard exudate aggregations cuffing the temporal border of the lesion. $\mathbf{d}$ Optical coherence tomographic section of the left eye; some degree of subfoveal subretinal fluid, inner retinal splitting, and high serous retinal detachment at the temporal retina. e Fluorescein angiography of the left eye, composite picture, and venous phase; the hypofluorescent lesion with stained border. $\mathbf{f}, \mathbf{g}$ Fluorescein angiography of the left eye and late venous phase; vascular changes and their staining at the border of the hypofluorescent lesion. 
implants were administered no satisfactory regression of the subretinal exudation was achieved (Fig. 2a). On B-mode ophthalmic ultrasound, there was an elevated mass-like lesion with a size of $6.5 \mathrm{~mm}$ in basal diameter and $3.7 \mathrm{~mm}$ in height. The tumor had medium to high internal reflectivity without any choroidal excavation (Fig. 2b).On MRI, the mass was relatively hyperintense with respect to the vitreous in the T1-weighted image (Fig. 2c). As no satisfactory regression of subretinal exudation could be obtained, a CyberKnife robotic controlled SRS was planned after reviewing the treatment alternatives with the patient and his family.

\section{CyberKnife SRS and the Clinical Course}

The patient underwent a $1.5 \mathrm{~T}$ planning MRI (General Electric). Two sequences are obtained consisting of a 3D 1-mm isotropic T2 series, and a gadolinium-enhanced 3D 1-mm T1 isotropic series. Retrobulbar block anesthesia was performed for fixation of the globe during simulation CT and CyberKnife SRS treatment. Simulation CT was acquired with a 1-mm thickness.

In the planning system (Multiplan version 4.6, Accuray, Sunnyvale, CA, USA), CT and both MRI sequences are manually co-registered using the insertion and the optic nerve and lens as principal landmarks (Fig. 2d). The gross lesion volume was segmented using both MRI sequences and retinal location. The dimensions of the contoured volume were checked in relation to those measured on ophthalmic MRI scan. A $1 \mathrm{~mm}$ margin was added to create planning target volume (PTV). Organs at risk contoured including lens, optic nerve, and perifoveal area. The optic nerve of the treated eye was contoured with a small 1-2 mm gap at the nerve insertion in the globe. A shell structure was created $2 \mathrm{~mm}$ beyond the PTV. The collimator
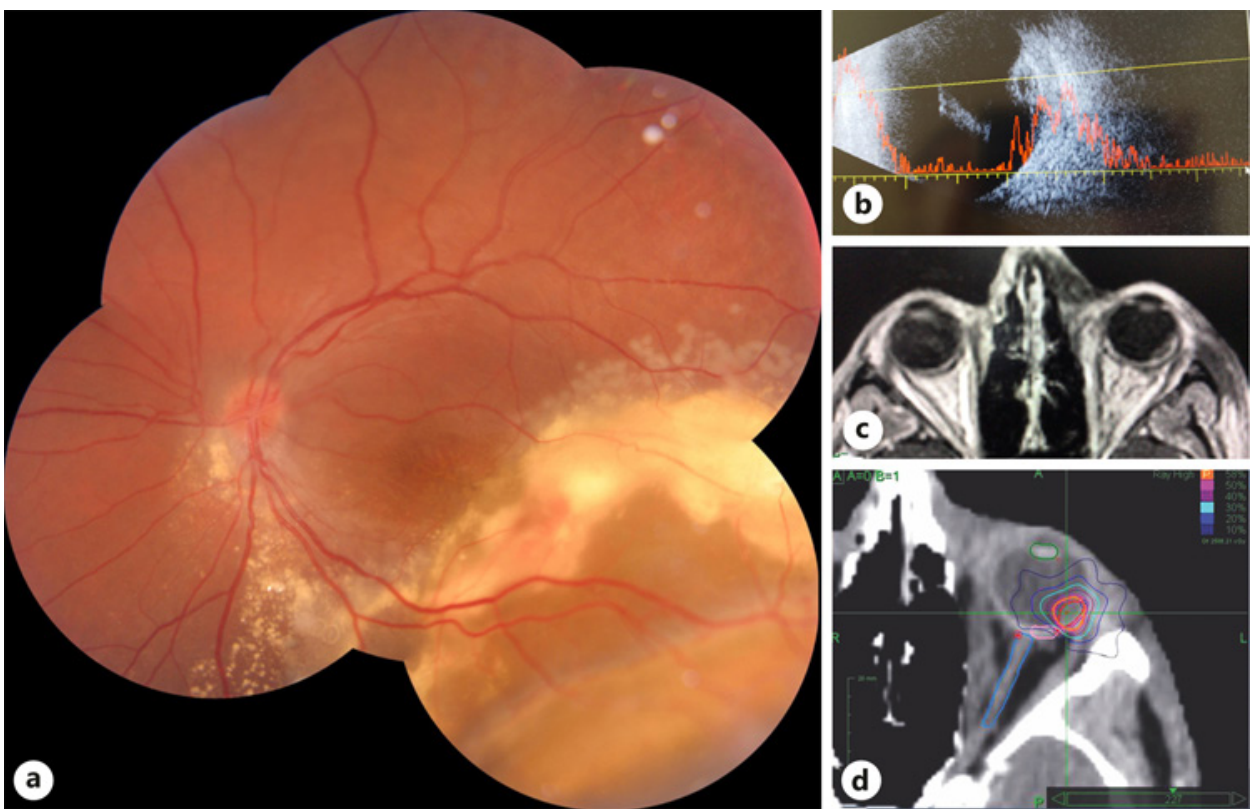

Fig. 2. Left eye (just prior to CyberKnife session). a Composite color fundus picture, temporally elevated mass-like lesion, surrounding exudative detachment and previous laser burns. b A and B scan ultrasonographic images of the retinal mass exhibiting a medium internal reflectivity consistent with a proliferative tumor. c MRI picture of the lesion in T1-weighted image. $\mathbf{d}$ Computerized tomographic appearance of the lesion involving the CyberKnife treatment plan protecting the optic nerve, lens, and the fovea. 
size is selected as $5 \mathrm{~mm}$. The plan is optimized so that the PTV is covered by at least $95 \%$ of the prescription dose with the $58 \%$ isodose volume. The conformity index (nCI) was 1.45 . The homogeneity index was 1.72.1,500 cGy in 1 fraction was prescribed to PTV, and the maximum doses to the lens and optic nerve of the treated eye were 87.96 and 213.97 cGy, respectively.

The patient was then immobilized in a supine position within a thermoplastic mask. The patient in this report was treated with 94 noncoplanar beams with a single 5-mm collimator. Original SRS dose was calculated by tissue heterogeneity using a ray-tracing algorithm. The maximum dose to the PTV was 2,586 cGy, and 8,542 MU was required to deliver the planned treatment. With our 1,000 MU/min CyberKnife VSI, the treatment time lasted for $30 \mathrm{~min}$. Twenty months after the CyberKnife SRS, visual acuity remained stable with significant resorption of the hard exudates along with regression in tumor thickness located at the temporal retina, and the lesion could be observed barely by the ultrasonography (Fig. 3a-d).

\section{Discussion}

VPTR is a subgroup of primary retinal telangiectasia which usually represents an elevated pink/yellow mass located in the periphery of the retina, with quite amount of retinal and subretinal exudation $[1,2]$. The lesion usually involves the inferotemporal retinal periphery, as in our case, but tumors can also be detected in other retinal locations [2]. Macular edema, exudation, and serous retinal detachment may contribute to the visual decline. The lesions in the earlier series were idiopathic in $74 \%$ of cases, with the remainder secondary to other conditions including XLRS.
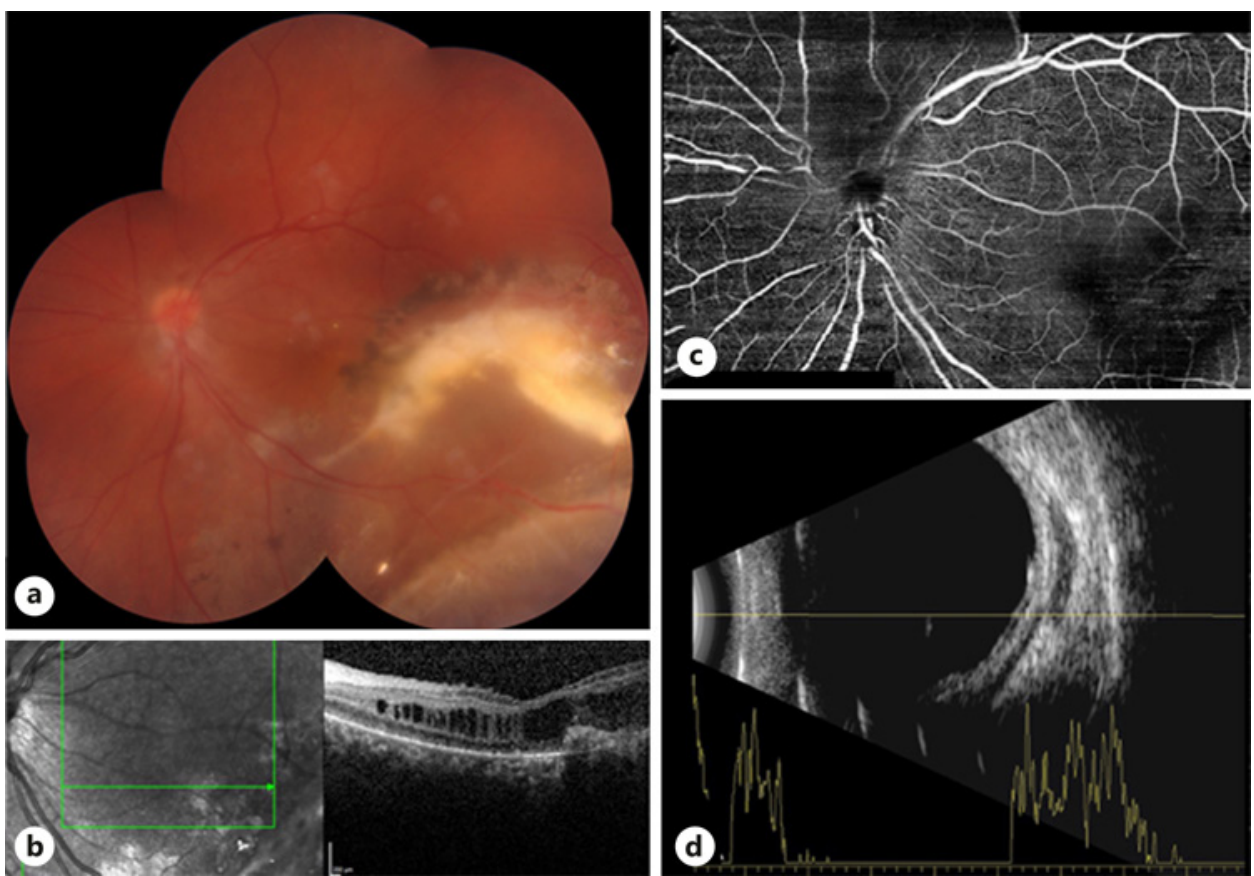

Fig. 3. Left eye (twenty months after the CyberKnife session). a Composite color fundus picture, partially regressed hard exudate cuff around the regressed lesion. b Optical coherence tomographic section and inner retinal splitting with hyperreflective hard exudate aggregates. c Composite optical coherence tomographic angiography of the posterior pole. $\mathbf{d}$ A and B scan ultrasonographic appearance of the fundus with the barely visible lesion. 
The response to conventional treatment is highly unpredictable [1, 2]. Anti-VEGF therapy is usually ineffective in these cases [5]. Shields et al. [2] tried to treat active VPTR using transscleral cryotherapy; however, the side effects include scleral thinning with discoloration and vitreous hemorrhage. Intravitreal dexamethasone implants may preventleakage from dysplastic retinal telangiectatic vessels and provide resorption of the fluid [6, 7]. Patel et al. [3] described a 17-year-old boy with XLRS and retinal detachment together with a VPTR. The patient was treated with pars plana vitrectomy, endolaser photocoagulation, subtenon's triamcinolone injection, and successive intravitreal bevacizumab injections. His vision improved and significant resolution of the leakage was observed as demonstrated by fluorescein angiography. However, intravitreal dexamethasone implant had only a limited effect in our case.

Ruthenium-106 and iodine-125 plaque radiotherapies for VPTR lesions have been reported with significant regression of tumors previously $[8,9]$. As our case had an inadequate response to intravitreal dexamethasone administrations and we have previous experience with CyberKnife SRS for other types of ocular tumors, we decided to perform CyberKnife SRS in the present case. CyberKnife SRS treatments were used with success for ocular melanoma [10,11], choroidal hemangioma [12], and optic nerve sheath meningioma [13] in the literature.

\section{Conclusion}

To our knowledge, the presented case is the first reported case of VPTR associated with XLRS treated with CyberKnife SRS. In VPTR, the classical therapeutic strategies are aimed to reduce the growth of the lesion, retinal exudation, and improve vision. Our case indicates that when primary treatment methods fail, CyberKnife SRS may achieve tumor regression and satisfactory clinical outcomes in VPTR.

\section{Statement of Ethics}

Written informed consent was obtained from the patient's parents for publication of this case report and any accompanying image.

\section{Conflict of Interest Statement}

All authors have no financial disclosures.

\section{Funding Sources}

There was no funding or grant support.

\section{Author Contributions}

S.C.I.: involved in writing of the manuscript. A.Y. and A.O.S.: involved in the ophthalmological examination and editing of the manuscript. M.T. and K.O.: involved in the radiotherapy, follow-up, and editing of the manuscript. All authors attest that they meet the current ICMJE criteria for authorship.

\section{Karger'}




\section{References}

1 Poole Perry LJ, Jakobiec FA, Zakka FR, Reichel E, Herwig MC, Perry A, et al. Reactive retinal astrocytic tumors (so-called vasoproliferative tumors): histopathologic, immunohistochemical, and genetic studies of four cases. Am J Ophthalmol. 2013;155(3):593-e1.

2 Shields CL, Shields JA, Barrett J, De Potter P. Vasoproliferative tumors of the ocular fundus. Classification and clinical manifestations in 103 patients. Arch Ophthalmol. 1995;113(5):615-23.

3 Patel NA, Laura D, Tran KD, Chang S, Barile G, Berrocal AM. Retinal vasproliferative tumor in a case of X-linked retinoschisis detachment. Am J Ophthalmol Case Rep. 2018;9:48-50.

4 Molday RS, Kellner U, Weber BH. X-linked juvenile retinoschisis: clinical diagnosis, genetic analysis, and molecular mechanisms. Prog Retin Eye Res. 2012;31(3):195-212.

5 Kenawy N, Groenwald C, Damato B. Treatment of a vasoproliferative tumour with intravitreal bevacizumab (Avastin). Eye. 2007;21(6):893-4.

6 Shields CL, Kaliki S, Al-Dahmash S, Rojanaporn D, Shukla SY, Reilly B, et al. Retinal vasoproliferative tumors: comparative clinical features of primary vs secondary tumors in 334 cases. JAMA Ophthalmol. 2013;131(3): 328-34.

7 Saatci AO, Doruk HC, Yaman A. Intravitreal dexamethasone implant (ozurdex) in coats' disease. Case Rep Ophthalmol. 2013;4:122-8.

8 Anastassiou G, Bornfeld N, Schueler AO, Schilling H, Weber S, Fluehs D, et al. Ruthenium-106 plaque brachytherapy for symptomatic vasoproliferative tumours of the retina. Br J Ophthalmol. 2006;90(4):447-50.

9 Cohen VM, Shields CL, Demirci H, Shields JA. Iodine I 125 plaque radiotherapy for vasoproliferative tumors of the retina in 30 eyes. Arch Ophthalmol. 1960;126(9):1245-51.

10 Eibl-Lindner K, Fürweger C, Nentwich M, Foerster P, Wowra B, Schaller U, et al. Robotic radiosurgery for the treatment of medium and large uveal melanoma. Melanoma Res. 2016;26(1):51-7.

11 Daftari IK, Petti PL, Larson DA, O'Brien JM, Phillips TL. A noninvasive eye fixation monitoring system for CyberKnife radiotherapy of choroidal and orbital tumors. Med Phys. 2009;36(3):719-24.

12 Agarwal A, Raghavan V, Rathnadevi R, Rishi P. Treatment of circumscribed choroidal hemangioma using CyberKnife: a viable alternative. Indian J Ophthalmol. 2019;67(5):704.

13 Bloch O, Sun M, Kaur G, Barani IJ, Parsa AT. Fractionated radiotherapy for optic nerve sheath meningiomas. J Clin Neurosci. 2012;19(9):1210-5. 